\title{
Les écoles d'Ontario ou le dilemme des conservateurs québécois : confrontation des principes nationalistes et de la réalité politique
}

\section{Nelson Michaud}

Volume 49, numéro 3, hiver 1996

URI : https://id.erudit.org/iderudit/305448ar

DOI : https://doi.org/10.7202/305448ar

Aller au sommaire du numéro

\section{Éditeur(s)}

Institut d'histoire de l'Amérique française

ISSN

0035-2357 (imprimé)

1492-1383 (numérique)

Découvrir la revue

Citer cet article

Michaud, N. (1996). Les écoles d'Ontario ou le dilemme des conservateurs québécois : confrontation des principes nationalistes et de la réalité politique. Revue d'histoire de l'Amérique française, 49(3), 395-417.

https://doi.org/10.7202/305448ar

\section{Résumé de l'article}

1916 : le premier ministre canadien Robert Laird Borden reçoit la demande pressante des Franco-Ontariens d'intervenir en vue de protéger leurs droits culturels et linguistiques menacés par la loi adoptée par la législature de Queen's Park et basée sur le fameux Règlement XVII promulgué en 1912. Le chef du gouvernement canadien se trouve alors devant un double dilemme : il doit à la fois évaluer la question sur un plan légaliste - l'intervention fédérale sur une question de juridiction provinciale - et composer avec un Cabinet où siègent des ministres francophones pour la plupart élus sous la bannière nationaliste. Leur credo essentiellement autonomiste cherche à faire du Canada un pays émancipé au sein de l'Empire, mais surtout à affirmer le caractère bi-ethnique de la nation; les défenseurs du nouveau Canada se disent alors " fidèles à l'esprit de Macdonald et de Cartier ». Face à la menace d'assimilation ressentie par leurs compatriotes d'outre-Outaouais, que feront ces députés-ministres conservateurs-nationalistes qui ont accédé au Cabinet Borden? Tenteront-ils d'intervenir dans le dossier malgré le fait qu'il s'agisse d'une question de juridiction exclusivement provinciale? Quelle attitude prendra le chef du gouvernement? La réponse à ces questions nous est ici révélée sous un jour inédit grâce à l'analyse des papiers personnels oubliés de l'un des acteurs de premier plan dans ce débat, le ministre Ésioff-Léon Patenaude. Plus de soixante-quinze ans plus tard, ces papiers nous livrent les éléments de compréhension nécessaires à une interprétation renouvelée de l'intervention potentielle du gouvernement fédéral dans la solution d'un problème qui a profondément marqué les Franco-Ontariens.
Tous droits réservés @ Institut d'histoire de l'Amérique française, 1996

Ce document est protégé par la loi sur le droit d'auteur. L'utilisation des services d'Érudit (y compris la reproduction) est assujettie à sa politique d'utilisation que vous pouvez consulter en ligne.

https://apropos.erudit.org/fr/usagers/politique-dutilisation/ 


\title{
LES ÉCOLES D'ONTARIO OU LE DILEMME DES CONSERVATEURS QUÉBÉCOIS: CONFRONTATION DES PRINCIPES NATIONALISTES ET DE LA RÉALITÉ POLITIQUE ${ }^{1}$
}

\author{
NELSON MICHAUD \\ Université Laval
}

\section{RÉSUMÉ}

1916: le premier ministre canadien Robert Laird Borden reçoit la demande pressante des Franco-Ontariens d'intervenir en vue de protéger leurs droits culturels et linguistiques menacés par la loi adoptée par la législature de Queen's Park et basée sur le fameux Règlement XVII promulgué en 1912. Le chef du gouvernement canadien se trouve alors devant un double dilemme: il doit à la fois évaluer la question sur un plan légaliste - l'intervention fédérale sur une question de juridiction provinciale et composer avec un Cabinet où siègent des ministres francophones pour la plupart élus sous la bannière nationaliste. Leur credo essentiellement autonomiste cherche à faire du Canada un pays émancipé au sein de l'Empire, mais surtout à affirmer le caractère bi-ethnique de la nation; les défenseurs du nouveau Canada se disent alors «fidèles à l'esprit de Macdonald et de Cartier». Face à la menace d'assimilation ressentie par leurs compatriotes d'outre-Outaouais, que feront ces députés-ministres conservateurs-nationalistes qui ont accédé au Cabinet Borden? Tenteront-ils d'intervenir dans le dossier malgré le fait qu'il s'agisse d'une question de juridiction exclusivement provinciale? Quelle attitude prendra le chef du gouvernement? La réponse à ces questions nous est ici révélée sous un jour inédit grâce à l'analyse des papiers personnels oubliés de l'un des acteurs de premier plan dans ce débat, le ministre Ésioff-Léon Patenaude. Plus de soixante-quinze ans plus tard, ces papiers nous livrent les éléments de compréhension nécessaires à une interprétation renouvelée de l'intervention potentielle du gouvernement fédéral dans la solution d'un problème qui a profondément marqué les Franco-Ontariens.

\section{ABSTRACT}

In 1916, Canadian Prime Minister Robert Laird Borden received a pressing request from Franco-Ontarians demanding that the federal government intervene to

1. Nous tenons à remercier le professeur Réal Bélanger du département d'histoire de l'Université Laval qui a dirigé la recherche à la base de cet article et qui nous a de plus fait part de judicieux commentaires à la lecture d'une première version de ce texte. Notre gratitude va aussi aux évaluateurs de la Revue d'histoire de l'Amérique française pour leurs remarques appropriées.

RHAF, vol. 49, n 3, hiver 1996 
protect their culture, religion, and language. These were threatened by a bill adopted by the Ontario legislature confirming the infamous By-law 17. The Canadian head of government faced a double dilemma: in addition to weighing the legal implications of a federal incursion into provincial jurisdiction, he was forced to deal with a Cabinet composed in part of French Canadians elected under the nationaliste banner. These ministers preached the gospel of an autonomous Canada within the British Empire while affirming the bi-ethnic character of the nation. This, they argued, was "true to the spirit of Macdonald and Cartier». Their response (and Borden's) to a situation that raised the issues of the possible assimilation of their Franco-Ontarian compatriots and federal interference in provincial jurisdiction is the subject of this article. The analysis of the forgotten personal papers of one of the ministers, EsioffLéon Patenaude, affords us a new reading of the federal government's potential intervention in a matter that profoundly marked the whole Franco-Ontarian community.

L'analyse des événements se rapportant à la lutte des FrancoOntariens en vue de l'obtention de certains droits linguistiques a déjà retenu l'attention de plusieurs historiens et historiennes. Plus particulièrement, le fameux épisode du Règlement XVII au sujet de l'enseignement en français, constitue un élément assez important dans l'évolution de la fédération canadienne pour que de nombreuses études en relatent les principaux éléments, page marquante des relations entre anglophones et francophones à un moment où le contexte de la Première Guerre mondiale amène les deux groupes à considérer maintes situations selon des positions opposées. Nous ne reprendrons donc pas ici ce que d'autres ont déjà adéquatement cerné2; notre propos consistera davantage à analyser ces événements sous un angle négligé jusqu'à ce jour. Nous aborderons la question sous l'angle de la fédération plutôt que sous l'angle strictement provincial, puisqu'il s'agit aussi d'un mornent charnière où le pays, vieux d'à peine un demi-siècle, a dû s'interroger sur l'élément constitutionnel à privilégier: bien que la question relevât essentiellement du gouvernement

2. Pour connaître les détails de l'affaire, on peut consulter, en plus des ouvrages cités ailleurs dans le texte: Lionel Groulx, L'enseignement français au Canada, II: Les écoles des minorités (Montréal, Granger Frères, 1933), 194-239; Margaret Prang, «Clerics, Politicians and the Bilingual Schools Issue in Ontario, 1910-1917», R. C. Brown, Minorities, Schools and Politics (Toronto, McClelland \& Stewart [1974]), 85-111; Marilyn Barker, «The Ontario Bilingual Schools Issue: Sources of Conflict», Idem; Albert Plante, Les écoles bilingues d'Ontario (Sudbury, La Société historique du Nouvel-Ontario, 1954), 47 p.; et André Lalonde, Le Règlement XVII et ses répercussions sur le Nouvel-Ontario (Sudbury, La Société historique du Nouvel-Ontario, 1965), 71 p. Mason Wade, malgré quelques imprécisions, brosse un bon tableau de la question en la situant dans le contexte politique global de l'époque dans Les Canadiens français de 1760 à nos jours, II: 1911-1963 ([Ottawa], Le Cercle du Livre de France [1963]), 85-106. Quant à Georgette Lamoureux, elle en trace une chronique intéressante et adéquatement illustrée dans son Ottawa, 1900-1926, et sa population canadienne-française ([Ottawa], G. Lamoureux, 1984). 
de Queen's Park, n'y avait-il pas là matière à intervention fédérale au nom de la protection de la minorité en faisant respecter l'article 93 de l'Acte de l'Amérique du Nord britannique? ? Devait-on privilégier l'autonomie des provinces ou prendre plus fermement position que ne l'avait fait le premier ministre libéral Wilfrid Laurier sur la question des écoles catholiques du Manitoba à l'automne 1896, puis sur celle des écoles des nouvelles provinces de Saskatchewan et d'Alberta à l'hiver $1905^{4}$ ?

Notre analyse est donc établie à partir du point de vue d'Ottawa. Elle nous amènera d'abord à brosser un tableau de la situation et à dresser un portrait de l'équipe fédérale en place. Elle nous permettra, par la suite, de scruter les interventions gouvernementales au plan de la stratégie à planifier, du double problème qui se pose (protection des minorités $v s$ autonomie provinciale), de la solution privilégiée et de la logique l'ayant amenée. Ces éléments nous feront découvrir que ce qui explique le mieux l'attitude du premier ministre fédéral conservateur, Robert Laird Borden, semble se trouver dans un dosage tout politique entre le respect manifesté pour les institutions provinciales et celui réservé à son électorat principal, anglophone et protestant. Par ailleurs, nous apporterons un éclairage inédit à la question en répondant à une question sous-jacente au problème: à un moment où il existe pourtant, selon l'historien Pierre Savard, «un mouvement de solidarité sans précédent entre Franco-Ontariens et Franco-Québécois ${ }^{5}$ », l'unité des ministres canadiens-français du Cabinet est-elle aussi forte dans les faits qu'en apparence, comme le prétendent plusieurs historiens? Il semble plutôt que pour certains ministres, l'argument d'autonomie provinciale ait eu son effet alors que pour d'autres, c'est davantage la nécessité de ne pas attiser une situation explosive qui ait prévalu,

3. Cet article de la constitution canadienne garantit en quelque sorte le statu quo ante quant aux écoles séparées. On aurait pu penser aussi à une évocation de l'article 90 touchant le pouvoir de désaveu, moyen très à la mode dans les premières années de la Confédération si l'on en croit l'analyse qu'en fait le constitutionnaliste Gil Rémillard (Le fédéralisme canadien. Éléments constitutionnels de formation et d'évolution (Montréal, Québec/Amérique, 1980), 147). Toutefois, ce pouvoir s'exerce au moment de l'adoption d'une loi; puisqu'il n'existe pas de clause rétroactive à ce pouvoir, il semble difficile de l'évoquer a posteriori.

4. Ces épisodes sont très bien documentés et pratiquement tous les ouvrages traitant de la carrière politique de Laurier peuvent nous fournir les détails à cet égard. Pour n'en citer que deux, mentionnons celui de Richard Jones, Vers une hégémonie libérale; aperçu de la politique canadienne de Laurier à King (s.1., Librairie des Presses de l'Université Laval, 1980), 27-44 pour les écoles du Manitoba et celui de Réal Bélanger, Wilfrid Laurier; quand la politique devient passion (Québec/Montréal, Les Presses de l'Université Laval/Les entreprises RadioCanada, 1986), 187-199 et 209-214 pour les écoles du Manitoba, et 295-306 pour les écoles de l'Alberta et de la Saskatchewan.

5. Pierre Savard, «Relations avec le Québec», Cornelius Jaenen, dir., Les FrancoOntariens (Ottawa, Presses de l'Université d'Ottawa [1993]), 235. 
ceux-ci étant soucieux de ne pas provoquer de blessures irréparables au sein d'un pays trop jeune, pourtant conçu dans un esprit de respect «des deux peuples fondateurs».

Cette double filiation nous est apparue à la lumière des recherches que nous avons conduites en rapport avec l'étude de l'idéologie nationaliste et de la carrière du ministre fédéral montréalais ÉsioffLéon Patenaude. Nous avons notamment retracé les papiers personnels du politicien, témoins oubliés que nul historien avant nous n'avait interrogés. Ce point de vue tout à fait inédit nous permettra de jeter un regard neuf sur un des moments les plus mouvementés de l'histoire canadienne contemporaine.

\section{UNE MINORITÉ, UN DÉFI}

Considérons tout d'abord le problème sur un plan général. La minorité francophone de la province d'Ontario réclame des institutions où ses enfants pourront étudier en français et être éduqués selon les préceptes de la religion catholique. À la suite d'une enquête qu'il a commandée, le gouvernement conservateur ontarien de J. P. Whitney lui répond par la «circulaire d'instructions $\mathrm{n}^{\circ} 17$ », mieux connue sous le nom de «Règlement XVII». Promulgué en 1912, amendé en 1913 et confirmé par une loi en 1915, le règlement interdit la fondation de nouvelles écoles bilingues, restreint aux premières années l'enseignement de la langue française et son utilisation comme moyen de communication, rend obligatoire l'apprentissage de l'anglais dès l'entrée à l'école et soumet les écoles anglaises/françaises à un double système d'inspection. Les francophones sont stupéfaits: plutôt que de répondre à leurs demandes, la mesure vise à calmer les craintes des anglophones qui se sentent menacés par un accroissement démographique important chez les francophones ${ }^{6}$. En fait, ces moyens draconiens autant que le ton sans équivoque du texte provoquent les Franco-Ontariens; bien vite la résistance s'organise dans les villages à prédominance franco-ontarienne dans le sud-ouest, fief de l'évêque Fallon, Canadien anglais d'origine irlandaise; bien que pour des raisons différentes, il se veut aussi anti-Canadiens français que les plus purs orangistes. Le mouvement est aussi ressenti dans le NouvelOntario (aujourd'hui, le Nord-Ontario) de même que dans l'est ontarien où la vague atteint la capitale nationale qui devient le centre des luttes. Dès 1912, le Conseil des écoles séparées d'Ottawa refuse

6. Selon le recensement officiel du Canada de 1911, la population francophone de l'Ontario représente 202442 personnes. Plus que le nombre absolu, c'est la proportion qui inquiète les anglophones: de $2,4 \%$ à la veille de la Confédération (recensement de 1861), les francophones comptent maintenant pour $8 \%$ du total des Ontariens en 1911, soit plus du triple en à peine deux générations. 
carrément d'appliquer le Règlement; en 1915, deux institutrices, les sœurs Diane et Béatrice Desloges, ouvrent une école parallèle après avoir été expulsées de l'école Guigues; des manifestations ont lieu et des femmes armées de longues épingles à chapeau, les «gardiennes d'écoles», empêchent les commissaires de faire appliquer le règlement. Après plus de trois années de vains efforts pour faire bouger le gouvernement provincial, les Franco-Ontariens se tournent vers le gouvernement fédéral: en février 1916, le curé de la cathédrale d'Ottawa, l'abbé Myrand, se rend chez le premier ministre Borden à la tête d'une délégation de 5000 Canadiens français pour le saisir du dossier et demander à son gouvernement d'intervenir? ${ }^{7}$. Devant l'ampleur du problème et son débordement sur la scène nationale, les politiciens fédéraux sont appelés à prendre position. Que feront-ils?

\section{UNE ÉQUIPE FÉDÉRALE AUX ACCENTS NATIONALISTES}

Pour bien comprendre l'action qui sera mise de l'avant par l'équipe gouvernementale, il est important de réaliser qu'elle est composée, en partie, de politiciens se déclarant autonomistes face à l'Empire et défenseurs du respect des «deux peuples fondateurs», dans l'esprit des Pères de la Confédération. Pour la plupart élus en 1911 en s'opposant au projet de marine militaire du premier ministre Laurier ${ }^{8}$, ces politiciens d'une nouvelle vague sont reconnus par Le Devoir sous le vocable de «conservateurs nationalistes ${ }^{9} »$. D'abord menés par F. D. Monk, ces hommes politiques composent l'essentiel du caucus conservateur québécois, et lorsque la question des écoles ontariennes est brûlante d'actualité, deux des trois ministres québécois francophones entourant Borden sont d'allégeance nationaliste ${ }^{10}$; il s'agit de Pierre-

7. «Les Canadiens français chez M. Borden», Le Devoir, 25 février 1916, 1.

8. Canadienne en temps de paix, cette marine deviendrait britannique en temps de guerre. Pour bien comprendre l'incidence de cette proposition, rappelons que nous sommes ici au lendemain de la guerre des Boers et à la veille de la Première Guerre mondiale.

9. Le Devoir, 19 août 1913,1.

10. Dans son article «The French Lieutenant in Ottawa» (R. Kenneth Carty et W. Peter Ward, National Politics and Community in Canada (Vancouver, University of British Columbia Press, 1986), 192), l'historien John English prétend qu'avec le départ de Monk en 1912, l'alliance conservatrice-nationaliste n'existe plus. Des études menées par d'autres historiens tels Réal Bélanger (L'impossible défi. Albert Sévigny et les Conservateurs fédéraux (1902-1918) (Sainte-Foy, Presses de l'Université Laval, 1983), 365 p.), Michèle Jean (Pierre-Édouard Blondin: la lourde hypothèque d'un passé nationaliste (1908-1921), mémoire de maîtrise, Université Laval, 1985, 122 p.), François Béland (F.D. Monk, le parti conservateur fédéral et l'idée d'un Canada pour les Canadiens (1896-1914), mémoire de maîtrise, Université Laval, 1986, 171 p.), Nelson Michaud (La carrière politique fédérale d'Ésioff-Léon Patenaude (19151926) ou l'affirmation continue du nationalisme canadien, mémoire de maitrise, Université Laval, 1988, 249 p.) ou Danièle Goulet (Louis-Philippe Pelletier: un exemple du douloureux mariage du mouvement nationaliste et du parti conservateur fédéral (1911-1914), mémoire de maîtrise, Université Laval, 1992), démontrent le contraire. 
Édouard Blondin, secrétaire d'État, et d'Ésioff-Léon Patenaude, ministre du Revenu de l'Intérieur récemment élu député d'Hochelaga lors d'un scrutin partiel ${ }^{11}$. Le troisième ministre du Québec est Tom Chase Casgrain, directeur général des Postes, un vétéran ayant repris du service en 1914 à la faveur d'une élection complémentaire dans la circonscription de Québec-Comté. Son idéologie est beaucoup plus près des valeurs impériales que ne l'est celle affichée et défendue par ses deux collègues.

Il est alors intéressant de suivre et d'analyser l'attitude de Borden, chef fédéral tory, vis-à-vis ses collègues du Québec à ce moment crucial des relations entre Canadiens anglophones et francophones. Ainsi, on constate que lorsque le premier ministre reçoit la visite de l'abbé Myrand, il est flanqué de Blondin et de Casgrain ${ }^{12}$. Où se trouve alors Patenaude? Aucun indice ne peut nous l'indiquer. Son absence est-elle due à des raisons personnelles ou a-t-elle été décidée par une instance supérieure? Encore là, le mystère demeure entier. Chose certaine, le premier ministre semble réticent à impliquer Patenaude dans le dossier. À preuve, cette insertion dans son journal personnel qui relate un entretien qu'il a eu avec Casgrain au sujet d'une rencontre au presbytère de l'abbé Myrand. Casgrain y a été invité mais il suggère au premier ministre d'y déléguer Patenaude. Borden affirme qu'il «debated permetting Patenaude to go but finally consented on condition that he would say nothing and not commit himself ${ }^{13}{ }^{3}$. On sent l'hésitation du leader tory: considère-t-il son ministre-recrue comme étant trop inexpérimenté? trop fougueux? ou trop nationaliste...? Craint-il que Patenaude, déjà reconnu comme un défenseur de «l'égalité des deux peuples fondateurs», ne compromette le gouvernement par une action précipitée? Borden ne le révèle pas. Tout au plus, savons-nous qu'au lendemain de la rencontre, c'est de nouveau Casgrain et non Patenaude qui fait rapport au premier ministre: selon ce que nous apprend le journal de Borden en date du 11 avril, Patenaude a joué le rôle qu'on lui a assigné, quoique sa présence à elle seule aurait réussi à troubler le chef libéral, Laurier,

11. Patenaude a été élu par acclamation le 15 octobre 1915. Malgré cette arrivée tardive sur la scène fédérale, Patenaude n'est pas un nouveau venu dans la famille nationaliste. C'est lui qui avait présidé la fameuse réunion de la Patinoire Ontario en 1910 et qui avait été l'organisateur d'Arthur Gilbert, premier député conservateur-nationaliste élu, ayant accédé à la Chambre des communes à la faveur de sa victoire lors d'une élection partielle tenue en 1910 dans Drummond-Arthabaska, comté de résidence de Laurier, alors premier ministre. Lorsque Borden lui offre un siège à Ottawa, Patenaude est, depuis 1908, député de Laprairie à la législature provinciale.

12. «Les Canadiens français chez M. Borden», Le Devoir, 25 février 1916, 1. 1916.

13. Archives publiques du Canada (APC), Fonds Borden, Journal personnel, 10 avril 
qui assistait à la rencontre ${ }^{14}$. Quant au contenu même des entretiens, la conclusion qu'en tire le ministre du Revenu de l'Intérieur se veut sans équivoque: «P. is sure that motion will be presented ${ }^{15}$.»

\section{UNE STRATÉGIE À PLANIFIER}

Le signal est donné. Selon l'analyse de Patenaude, les libéraux sont donc prêts à poser publiquement un geste d'appui à la cause des Franco-Ontariens. En fait, depuis plusieurs mois déjà, la question a suscité maintes réactions ${ }^{16}$ et il est effectivement très plausible que le tout débouche sur des gestes concrets, très bientôt. Les conservateurs doivent donc être prêts à réagir. Borden et son Cabinet analysent, soupèsent, planifient. Depuis quelques semaines, l'effervescence des débats sur le sujet donne lieu à des séances ministérielles mouvementées; l'imminence d'un développement ne fait qu'en accroître la fébrilité.

Dans ce climat de tension, les trois ministres canadiens-français ne demeurent pas passifs: par la voix de Casgrain, ils proposent à leurs collègues du Cabinet de couper l'herbe sous le pied des libéraux en faisant présenter une motion par les conservateurs ${ }^{17}$. Cette action relève directement d'un conseil que leur donne Thomas Chapais, personnage en vue du Parti conservateur qui avait déjà tenté une intervention auprès de Whitney, le premier ministre ontarien ${ }^{18}$. Le futur sénateur Chapais suggère aux ministres canadiens-français de ne pas «laisser à Laurier la gloire d'être le champion de la minorité et du respect des engagements ${ }^{19} \gg$. De plus, Chapais croit que le désaveu demandé par une éventuelle motion libérale n'est pas la solution idéale. Cette dénonciation serait génératrice d'une crise aiguë entre Ottawa et les provinces. Chapais considère donc l'avenue que préconiseront probablement les libéraux, aussi inefficace que dangereuse, car, outre les tensions créées, elle n'apporterait rien qui pourrait remédier au Règlement XVII, cause même du mal ${ }^{20}$.

14. Idem, 11 avril 1916.

15. Idem.

16. Dans son étude sur Paul-Émile Lamarche, le professeur Réal Bélanger en dresse un bon bilan. Voir Paul-Émile Lamarche. Le pays avant le parti (1904-1918) (Sainte-Foy, Presses de l'Université Laval, 1984), 285-310.

17. APC, Fonds Borden, Journal personnel, 7 juillet 1916.

18. Tel que le relatent Robert Choquette (Langue et religion. Histoire des conflits anglofrançais en Ontario (Ottawa, Éditions de l'Université d'Ottawa, 1977), 175) et Savard (op. cit., 236).

19. Archives privées de la compagnie Industrielle-Alliance (APA), Fonds Patenaude, boîte 1, chemise «Écoles d'Ontario III", Chapais à Casgrain, 3 avril 1916.

20. Selon Robert Rumilly, Histoire du Québec, XXI: Courcellette (Montréal, Montréal éditions, s.d.), 84. Pour plus de précisions sur les vues de Chapais, on peut consulter son article «La langue française dans les écoles d'Ontario» publié dans la Revue Canadienne et reproduit dans ses Discours et Conférences (Québec, Garneau, 1913), deuxième série, 399-402. 
Même si la rencontre au presbytère de l'abbé Myrand confirme ce que prévoyaient Casgrain, Blondin et Patenaude, le Cabinet ne semble pas convaincu de la nécessité de prendre les libéraux à revers. Ne se laissant pas rebuter par ce premier refus, les trois ministres canadiensfrançais reviennent à la charge. Cette fois, leur action est planifiée et concertée à un point tel qu'elle provoque presque une crise ministérielle. Dix jours après la réunion chez le curé d'Ottawa, les ministres canadiens-français signent un mémoire qu'ils adressent à Borden et à son Cabinet et dans lequel ils exposent leur solution au problème. Afin de faire accepter leur point de vue, ils menacent de démissionner si Borden et le Cabinet (d'où ils s'abstiennent de siéger pendant les pourparlers) rejettent leur document.

\section{PROTECTION DES MINORITÉS OU AUTONOMIE PROVINCIALE?}

Ce mémoire peut révéler des éléments fort intéressants pour notre étude. Tout d'abord, il nous apprend que les trois ministres travaillent de concert; les trois signent le document: ils sont donc solidaires dans leur action. Si Casgrain se retrouve le plus souvent à l'avant-scène, cela tient à son ancienneté, d'une part, et fort probablement aussi du fait qu'il est celui des trois que Borden considère comme le plus modéré, le plus conciliant; le triumvirat des ministres canadiensfrançais a donc avantage à le déléguer pour plaider sa cause, le premier ministre lui prêtant $a$ priori, une oreille plus attentive. À moins de preuve contraire, on peut considérer que les propos et gestes de Casgrain sont endossés par Blondin et par Patenaude.

Par ailleurs, en analysant le long préambule du mémoire, il est possible de percevoir ce qui motive l'action de Patenaude et de ses collègues: c'est le désir de voir évoluer et se développer un Canada uni composé des deux peuples fondateurs égaux et ce, dans l'esprit des Pères de la Confédération. Cette question de l'unité nationale se trouve à la base même de leur démarche. Animés par «a sincere and patriotic desire to see peace and harmony reign in our beloved country ${ }^{21}$ », les ministres disent vouloir mettre fin à une situation qu'ils qualifient de terrible: ils veulent apporter une solution «which will restore our beloved country to its normal $s^{2} a^{22} \gg$. Quant à la place réservée aux Canadiens français au pays, les trois politiciens la définissent historiquement et font remarquer que celle-ci fut consacrée par Sir John A. Macdonald lui-même lors du débat sur la Confédération en 1865: «The use of the French language, avait-il déclaré, forms one

21. APC, Fonds Borden, Casgrain, Blondin et Patenaude à Borden, 20 avril 1916.

22. Idem. 
of the principles upon which confederation should be established and its use as at present should be guaranteed by the Imperial $\mathrm{Act}^{23}$.» Le cadre de l'intervention des trois ministres peut donc clairement être établi.

Après avoir nettement souligné l'importance de la question et son incidence partout au pays où il y a des Canadiens français, et plus particulièrement au Québec, les ministres résument le problème complexe en des termes clairs, simples et concis:

The question to be solved may be postulated as follows: - In view of the natural law, the custom and practice observed in this country, the legislative enactments which from time to time have been brought to bear upon the subject and the general policy of the Empire, what is the status of the French language and what recognition is to be secured to it generally throughout Canada ${ }^{24}$ ?

La réponse à cette interrogation pourrait être donnée par un tribunal indépendant composé de citoyens canadiens, avancent-ils; mais devant la difficulté de trouver des gens sans préjugé sur le sujet, les ministres suggèrent une autre avenue. "To remove the matter entirely outside of the influences, prejudices and predilections of any body of men in Canada, we have thought that the King in his Privy Council would be a tribunal which would readily be accepted by everybody ${ }^{25}$.»

Bien qu'ils précisent qu'il s'agisse d'un comité spécial et non d'un comité judiciaire du Conseil privé, cet appel aux instances impériales a de quoi surprendre de la part de nationalistes. Eux-mêmes en sont conscients: «We are quite aware that the procedure indicated above is an extraordinary method of dealing with the question, but it must be admitted that the circumstances of the case are extraordinary ${ }^{26} . »$ L'analyse qu'ils font de ces circonstances renferme, au-delà des technicalités juridiques dont ces avocats sont bien conscients, la raison fondamentale justifiant pareille action: c'est encore au nom du Canada uni et dans le respect de ses deux peuples qu'ils agissent. "Almost one-third of the population of Canada is arrayed against the other two-

23. Idem.

24. Idem. À remarquer l'envergure donnée à la question. Il ne s'agit plus des écoles franco-ontariennes mais de la place du français au Canada. Deux raisons peuvent expliquer cette prise de position: le problème des écoles françaises et des écoles séparées a déjà suscité maints débats dans les Territoires, au Manitoba et au Nouveau-Brunswick. Un énoncé général de politique viserait à conclure le débat une fois pour toutes. Par ailleurs, le fait de ne pas discuter d'un cas précis et de ne pas aborder la question sous un angle purement légal vient aussi du contexte où se situe le problème à ce moment précis: les tribunaux étant déjà saisis du cas, il est impossible d'intervenir, l'affaire étant sub judice.

25. Idem.

26. Idem. 
thirds; an agitation reaching beyond the confines of the Province of Quebec and tending to interrupt for all time the good relations which should exist between all Canadians is stirring the country to its very depths ${ }^{27}$.» Face à un problème aussi délicat, la conclusion à laquelle ils en arrivent démontre clairement le but ultime de leur action: «Our ardent desire is, if possible, to put a stop to this terrible situa$\operatorname{tion}^{28} . »$

Dès la réception du document, le premier ministre consulte son état-major: Arthur Meighen, le Solliciteur général, et George Eulas Foster, le vice-premier ministre ${ }^{29}$. Le lendemain, Meighen soumet un projet de réponse à son chef avec qui il arrive à un consensus ${ }^{30}$. Par la suite, Borden consulte différents ministres, puis son Conseil au complet. Après plus de deux heures et demie de discussion, le Cabinet en vient à une entente ${ }^{31}$. Le premier ministre fait donc parvenir sa réponse aux trois ministres canadiens-français qui, refusant toujours de siéger, sont demeurés dans l'expectative. «In the observations [you made], my colleagues and I agree. I am compelled therefore with much regret to advise you that the course you urge cannot be taken ${ }^{32}$.» Pour justifier sa réponse, Borden considère le même contexte que Patenaude et ses acolytes. Il arrive toutefois à une conclusion différente de celle avancée par ses ministres.

Selon le chef conservateur, les tribunaux ne peuvent statuer sur le français et la place qui lui revient au Canada. Il croit aussi qu'aller chercher un avis à l'extérieur du pays pour des questions de juridiction interne constitue un dangereux précédent. De plus, il considère que ce serait une atteinte aux pouvoirs et à l'autonomie provinciale que de s'immiscer dans le dossier. Enfin, il affirme que, pour le bien et l'unité du pays, on ne peut se permettre de prendre un si grand risque: si l'avis obtenu est défavorable et abolit l'usage du français partout, que dira le Québec? Et, corollairement, si l'avis est favorable et oblige l'utilisation du français dans tous les services publics, quelle sera l'attitude des autres provinces?

Devant les arguments servis par Borden, on peut se demander si ses ministres canadiens-français ont agi sous l'impulsion du moment

27. Idem.

28. Idem.

29. APC, Fonds Borden, Journal Personnel, 22 avril 1916.

30. Idem, 23 avril 1916.

31. Idem, 24 avril 1916. Les ministres consultés en privé sont White, Rogers, Reid et Doherty.

32. APA, Fonds Patenaude, boîte II, filière B, dossier 4, Borden à Casgrain, Blondin et Patenaude, 24 avril 1916. 
en faisant leur déclaration ou s'ils y entrevoyaient plutôt un argument politique à utiliser, quitte à le négocier par la suite, ou encore s'ils avaient l'intention de faire jouer une entente secrète de quelque espèce avec le gouvernement provincial québécois alors dirigé par Lomer Gouin. Patenaude, ayant une réputation bien établie à la législature de Québec, aurait pu en effet mettre à profit ses «contacts» provinciaux en ce sens. Aucun indice ne nous permet d'apporter une réponse affirmative à ces questions. Ce qui semble cependant plus près de la réalité, c'est que le refus systématique exprimé par leur chef n'a pas l'heur de plaire aux trois ministres. Ils répliquent donc, réfutant les objections qu'on leur a posées. Ils réaffirment à Borden qu'en allant à Londres, il n'est pas question de présenter la cause devant un tribunal mais bien devant un conseil composé d'hommes insensibles aux préjugés qui influenceraient un tribunal canadien. Leur décision serait reçue ici avec respect et les ministres assurent même Borden du respect de la décision par le Québec. En outre, les ministres rassurent leur leader en affirmant que: «Herefore we have not appealed in vain to the justice, fairness and spirit of fairplay of the British Autorities ${ }^{33} . \gg$

La réplique à cette «foolish letter ${ }^{34} »$ ne laisse guère place à la discussion. Borden dit avoir déjà répondu à toutes les nouvelles objections qu'on lui pose. Il réitère même que s'il n'a rien proposé, c'est par respect pour l'autonomie provinciale ${ }^{35}$. Semblable réponse signifie donc que la discussion sur le sujet est close. Avant de faire parvenir sa réponse définitive, Borden convoque toutefois Casgrain qu'il trouve «in a condition of collapse. No nerve, no back bone [...] He said Patenaude in Montreal, return early Wednesday and would give answer by noon. Saw Blondin afterwards who says whole situation due to Casgrain's stupidity and neglect and lack of nerve ${ }^{36}$ ».

Le front commun des ministériels canadiens-français, pourtant si apparent l'après-midi même ${ }^{37}$, commence-t-il déjà à s'effriter? C'est ce que semble croire Foster. Dans son journal personnel en date du 25 avril, il nous en dit long sur sa perception de la situation, malgré la brièveté de l'insertion du jour: «The detachment of Quebec Ministers is in a quandary ${ }^{38}$.»

33. APC, Fonds Borden, Casgrain, Blondin et Patenaude à Borden, 25 avril 1916.

34. APC, Fonds Borden, Journal personnel, 25 avril 1916.

35. APA, Fonds Patenaude, boîte II, filière B, dossier 4 , Borden à Casgrain, Blondin et Patenaude, 26 avril 1916.

36. APC, Fonds Borden, Journal personnel, 25 avril 1916.

37. Idem, «No French Minister or member on our side in House Today».

38. APC, Fonds Foster, Journal personnel, 25 avril 1916. 
Borden, qui anticipait déjà depuis quelque temps une scission au sein de son parti dans l'éventualité d'un vote sur la question ${ }^{39}$, profite de ce déséquilibre, fait tout pour ouvrir la brèche et anéantir la solidarité animant ses trois ministres canadiens-français; il veut à tout prix les amener à épouser la position du gouvernement. Il saisit le gouverneur général de la question ${ }^{40}$ et celui-ci convoque aussitôt Casgrain à Rideau Hall; après un bref entretien, le vice-roi convainc le directeur général des Postes de demeurer dans le ministère.

Pour certains, comme Foster, le problème est définitivement réglé. «The blow is over, confie-t-il à son journal. After the answer to the ultimatum the weather cleared, and now bids fair along that quarter of the $s k y^{41}$.» Casgrain abandonne le plan conçu dans le mémoire mais ne semble pas considérer la partie comme irrémédiablement perdue. À une séance du Cabinet, le 9 mai, il revient à la charge avec l'idée d'une motion dont l'initiative reviendrait à l'équipe ministérielle. Il renforce sa stratégie en soulignant que même si la motion devait être battue, elle ne pourrait pas être considérée comme une motion de nonconfiance mettant en danger la survie du gouvernement ${ }^{42}$. De nouveau, l'idée ne recueille pas les appuis voulus, d'autant plus que depuis vingt-quatre heures, le Parlement est déjà saisi d'une motion semblable, présentée par le député libéral de Kamouraska, Ernest Lapointe.

\section{LE TRIOMPHE DE L'AUTONOMIE}

Pendant trois jours, les Communes discutent de cette proposition qui «invite respectueusement l'Assemblée législative de l'Ontario à faire en sorte qu'il ne soit pas porté atteinte au privilège des enfants d'origine française de recevoir leur éducation dans leur langue maternelle ${ }^{43} \gg$. Malgré les nombreuses rencontres officielles, les multiples tractations d'officines et les tactiques savamment orchestrées dont elle fait l'objet, la motion, dont la paternité revient vraisemblablement à Wilfrid Laurier, est rejetée par 107 voix contre $60^{44}$.

39. APC, Fonds Borden, Journal personnel, 7 avril 1916. Comme le soulignent implicitement Francis, Jones et Smith, si ce n'était pas l'élément francophone qui ruait dans les brancards, c'était certains éléments anglophones «peu sensibles» au fait français, pour utiliser un euphémisme. (Incident relaté dans Destinies. Canadian History Since Confederation (Toronto, Holt Rhinehart et Winston, 1992), 209.)

40. APC, Fonds Borden, Borden à l'aide-de-camp du gouverneur général, 25 avril 1916.

41. APC, Fonds Foster, Journal personnel, 26 avril 1916.

42. APC, Fonds Borden, Journal personnel, 9 mai 1916.

43. Journal des débats de la Chambre des communes du Canada, Session 1916, IV: 3781, 8 mai 1916 .

44. Paul Bernier dresse un excellent tableau de toute la préparation et de la stratégie entourant la présentation de la motion dans son étude, Ernest Lapointe, député de Kamouraska, 1904-1919 (La Pocatière, Société historique de la Côte-du-Sud, 1979), 121-133. 
Les libéraux de l'Ouest sonnent le clairon de la dissidence. Ils sont menés par John Wesley Dafoe, rédacteur en chef du Winnipeg Free Press, et par Frank Oliver, ex-ministre de l'Intérieur. Les conservateurs s'y opposent également, à l'exception de sept d'entre eux, tous députés francophones du Québec élus en 1911 sous la bannière conservatrice-nationaliste ${ }^{45}$. Les rangs des dissidents sont complétés par l'indépendant Paul-Émile Lamarche, lui aussi ancien disciple nationaliste. Il dira plus tard, parlant de son vote, qu'il a alors «agi par acte de conscience ${ }^{46}$ ».

C'est donc dire que les ministres canadiens-français ont voté contre la motion. Pendant le débat, c'est encore Casgrain qui prend la parole. Dans un discours bien articulé, ponctué d'attaques partisanes bien senties, le directeur général des Postes se plie à la discipline de parti, rallie les propos de son chef et fait un vibrant plaidoyer pour l'autonomie provinciale, invoquant l'intérêt particulier que le Québec peut avoir en cette matière. Exposant clairement sa position, et conséquemment celle de ses collègues, il affirme:

Je ne veux pas discuter le côté légal de la question; les tribunaux en sont saisis; j'en appelle à l'histoire et [...] je m'en remets à la générosité de la population ontarienne. [...] Je ne veux pas qu'un règlement ou un décret de la législature de l'Ontario soit sujet à censure de la part de la Chambre des communes. [...] Non seulement cette résolution est inutile, mais elle est dangereuse. Elle est grosse de menaces pour les autres provinces, et surtout pour la province de Québec. [...] Je ne voudrais pas que l'on pût croire que j'approuve en ce moment ce qui s'est passé au Manitoba ou dans l'Ontario, mais j'affirme que ce n'est pas ici le lieu de discuter de cette question ${ }^{47}$.

Et, conscient des pressions venant d'un Québec réclamant une intervention fédérale dans le dossier, il termine son discours en déclarant avec fermeté: «Je le sais parfaitement bien, je vais à l'encontre de l'opinion de la grande majorité de la population dans la province de Québec en prenant cette attitude; mais, après mûre réflexion, je suis convaincu que c'est là mon devoir ${ }^{48} .{ }^{\text {.» }}$ 'est sans

45. Contrairement à ce que prétend Bernier (op. cit., 133), ils sont sept et non neuf. Il s'agit de Honoré Achim (Labelle), Joseph-Arthur Barrette (Berthier), Adélard Bellemarre (Maskinongé), Herménégilde Boulay (Rimouski), Albéric Archie Mondou (Yamaska), Dr Eugène Paquet (L'Islet) et Joseph-H. Rainville (Chambly). Voir Journal des débats..., Session 1916, IV: 4000, 12 mai 1916.

46. C'est ce qu'il déclare lors de son discours d'adieux à ses électeurs lorsqu'il se retire de la politique. Rapporté dans «Le sang de la nation hypothéqué», Le Devoir, 2 octobre 1916, 1.

47. Journal des débats..., Session 1916, IV: 3882-3888, 10 mai 1916. Voir aussi «La question bilingue est discutée à la Chambre», La Patrie, 11 mai 1916, 6.

48. Journal des Débats..., loc. cit., 3887. 
aucun doute pour cette raison que Casgrain sent le besoin d'expliciter davantage sa pensée lors d'une assemblée publique quelques mois plus tard: «Nous avons jugé qu'il serait dangereux de permettre au gouvernement fédéral de faire la moindre incursion dans un champ qui est réservé aux seules législatures, précise-t-il. Une fois la porte ouverte, on ne saurait plus la fermer et notre vote était un avertissement solennel pour les temps à venir que nous résisterions jusqu'au bout à tout empiétement de ce côtée ${ }^{49} . »$

Malgré cette apparente conversion due à sa «mûre réflexion», Casgrain tente un dernier effort: à quelques heures du vote sur la motion Lapointe, il suggère que ce vote soit pris librement, sans tenir compte de la ligne de parti. Une fois de plus, la tentative échoue: il se heurte, comme toujours, à ses collègues anglophones ${ }^{50}$. Casgrain rentre donc définitivement dans les rangs et subordonne la grandeur des principes nationalistes aux exigences de la vie politique. Quant à Blondin, la cascade d'excuses qu'il déverse aux pieds de Borden à la suite de l'attitude de Casgrain et les félicitations chaleureuses qu'il offre au premier ministre après son discours sur la motion Lapointe ${ }^{51}$ nous prouvent sa soumission. Une seule pièce manque au casse-tête: Patenaude. Est-il demeuré solidaire de Casgrain et de Blondin jusqu'à l'abdication ou ses principes nationalistes ont-ils pris le dessus et guidé son action vers une autre direction?

\section{UNE SOLIDARITÉ FRAGILE}

Chose certaine, les arguments de Borden repris par Casgrain concernant l'autonomie provinciale et le risque pour l'autonomie nationale d'associer Londres au dossier peut sembler très acceptable à un défenseur du nationalisme canadien autonomiste: ces concepts étaient ceux-là mêmes défendus par la Ligue nationaliste. Voilà un premier élément qui pourrait faire croire à la soumission immédiate de Patenaude, à l'instar de ses collègues. De plus, les propos mêmes du politicien donnent une certaine crédibilité à cette hypothèse: le député d'Hochelaga prône un climat de non-confrontation et adopte une attitude favorisant la «fermeté dans la modération». Au Sénateur Philippe Landry qui a démissionné de la présidence de la Chambre haute afin de s'impliquer corps et âme dans la lutte des FrancoOntariens ${ }^{52}$, il livre un avertissement clair et sans équivoque:

49. «Le Gouvernement a sauvé l'autonomie de notre province», La Patrie, 2 octobre 1916, 9.

50. APC, Fonds Borden, Journal personnel, 10 mai 1916.

51. Idem.

52. Voir «La démission du président du Sénat», La Patrie, 2 juin 1916, 1, et «Pour la question bilingue», 6 juin 1916, 2. Dans sa lettre de démission au premier ministre, Landry 
Je dois vous dire immédiatement, que si par lutte, on entend l'agitation qui s'est poursuivie dans le cours de l'année dernière [1916] à Ottawa, et dans la province de Québec, j'y suis opposé de toutes mes forces, et je suis sincèrement convaincu que cette agitation compromet la cause au lieu de l'aider. J'ajoute, que si par lutte on entend l'action à la fois ferme et discrète, et le travail persévérant, je continuerai de la favoriser de toutes mes forces. Je me permettrai de vous dire [aussi] que la cause de nos compatriotes serait bien plus avancée, si on leur avait toujours conseillé d'agir avec modération et prudence, et de se faire des alliés et non des ennemis dans tous les groupes ${ }^{53}$.

Patenaude s'était d'ailleurs déjà prévalu de ces mêmes principes, à la suite des verdicts rendus et par le Conseil privé de Londres et par le pape Benoît $\mathrm{XV}^{54}$. Le plus haut tribunal civil ayant juridiction au pays, en appel d'une décision de la Cour suprême de l'Ontario, et l'autorité suprême de l'Église catholique, à la demande de vingt-et-un évêques et archevêques canadiens-français, se sont en effet tous deux prononcés en faveur d'une attitude de non-provocation, celle-là même qu'ont adoptée les conservateurs francophones; le ministre du Revenu en conclut: "Quand on veut la justice, il vaut toujours mieux consulter d'abord la raison, l'avenir. Et ce qui conduit au succès, c'est la fermeté et la modération dans les revendications ${ }^{55}$.»

somme ce dernier d'agir. «Vous ne pouvez pas invoquer la loi pour vous justifier: elle est en notre faveur. Elle nous donne le pouvoir absolu, sans contrôle de désavouer toute législation provinciale et de protéger ainsi toute minorité qui souffre sous le talon d'une majorité intolérante [...] Les législatures n'ont un pouvoir souverain dans l'espèce qu'à la condition de ne pas empiéter sur les droits des minorités. Et c'est parce que la législature d'Ontario a franchi les limites que lui assigne la constitution dans l'exercice de son droit exclusif que nous demandons au pouvoir exécutif fédéral d'intervenir et de refouler le flot de fanatisme qui déborde.» APC, Fonds Borden, Landry à Borden, 29 mai 1916. (Une copie de la lettre se trouve au APA, Fonds Patenaude, boîte II, chemise «Résignation de l'hon. P. Landry»). Quant à Borden qui «sous les circonstances [n'a] pas d'hésitation à accepter votre démission comme président du Sénat», il se contente de répondre laconiquement qu'il ne se sent «aucunement disposé à commenter vos déclarations parmi lesquelles s'en trouvent une grande partie qui sont à la fois, ni justes, ni bien fondées». Idem, Borden à Landry, 30 mai 1916.

53. APA, Fonds Patenaude, boîte I, chemise «École d'Ontario IV», Patenaude à Landry, 18 mai 1917.

54. Publiés au début de novembre 1916. L'arrêté du Conseil privé représente de plus un fait tournant pour les Franco-Ontariens puisqu'il déclare ultra vires la mise en tutelle du Conseil des écoles d'Ottawa par l'instauration de ce qui a été connu sous le nom de «la p'tite commission». Pour de plus amples détails au sujet de cet aspect de la question, n'ayant toutefois pas eu de répercussions particulières sur nos considérations au niveau fédéral, on peut consuiter avantageusement l'ouvrage de Robert Choquette, La foi gardienne de la langue en Ontario, 1900-1950 (Montréal, Éditions Bellarmin, 1983), 121. Voir aussi à ce sujet Victor Simon, Le Règlement XVII: sa mise en reuvre à travers l'Ontario, 1912-1927 (Sudbury, Société historique du Nouvel-Ontario, 1983), 30.

55. «M. Patenaude est vengé», Le Devoir, 6 novembre 1916, 2. Voir aussi «L'assemblée d'hier à St-Jean-Chrysostôme», La Patrie, 6 novembre 1916, 2. Il est intéressant de noter que 
Fermeté et modération apparaissent donc nettement comme le leitmotiv de l'action de Patenaude. Cette attitude signifie-t-elle pour autant la soumission et l'oubli des principes nationalistes? Selon les études publiées à ce jour par les historiens Robert Rumilly ${ }^{56}$, Robert Choquette $^{57}$, F. A. Walker ${ }^{58}$, Robert Craig Brown ${ }^{59}$ et Mason Wade ${ }^{60}$, il semblerait bien que ce soit le cas. Tous reprennent ce que rapportent les mémoires de Borden ${ }^{61}$ qui affirment que, à la suite de pourparlers serrés, Casgrain abandonne la lutte, les deux autres ministres à sa suite. Pour aussi «satisfaisante» qu'elle puisse apparaître, cette interprétation laisse beaucoup de questions sans réponse; ainsi, le but principal de l'action des trois ministres canadiens-français était-il véritablement celui de l'édification et de la reconnaissance d'un Canada uni et biculturel? Et si ce sont vraiment ces principes nationalistes qui sont à la base de leurs actes, pourquoi n'ont-ils pas été jusqu'au bout et n'ont-ils pas démissionné comme ils ont menacé de le faire? Les politiciens ont-ils agi seuls ou ont-ils impliqué d'autres personnes dans leur démarche? Quant à Patenaude, est-il constamment demeuré solidaire de ses deux collègues? Encore ici, il faut fouiller plus à fond pour élucider ces énigmes jusqu'à ce jour irrésolues.

\section{UN NOUVEL ÉCLAIRAGE}

Grâce aux recherches que nous avons menées dans le fonds inédit de Patenaude, il nous est désormais possible de répondre à ces questions et de connaître le sens profond des démarches entreprises par les ministres canadiens-français, voire leurs réflexions. Cette information nous a été fournie grâce à son compte rendu des événements, sorte de journal qui relate et analyse les faits entourant l'implication des trois ministres canadiens-français dans le dossier ${ }^{62}$. Ce

c'est ici la seule référence que Patenaude fait à l'aspect religieux du conflit, fait étonnant si l'on considère l'importance de cette dimension, autant d'une manière générale dans le credo nationaliste canadien de Bourassa, que d'une manière plus particulière dans les revendications franco-ontariennes. Il n'existe d'autres explications à cet écart que la ligne de conduite généralement laïque observée par Patenaude tout au long de sa carrière politique.

56. Robert Rumilly, Histoire du Québec, XXI: 88.

57. Robert Choquette, Langue et religion..., 203.

58. F. A. Walker, Catholic Education and Politics in Ontario (Toronto, Thomas Nelson \& Sons, 1964).

59. Robert Craig Brown, Robert Laird Borden: a biography, II: 1914-1937 (Toronto, MacMillan of Canada [1980]), 51-52.

60. Mason Wade, Les Canadiens français..., II: 101.

61. Henry Borden, ed., Robert Laird Borden, his Memoirs, II: 1916-1920 ([Toronto], McClelland \& Stewart [1969]), 21-23. On y donne le texte intégral du mémoire et de la réponse de Borden.

62. APA, Fonds Patenaude, boîte II, filière B, dossier 4, «Notes re: Statut du français au Canada et mémoire des ministres de langue française au premier ministre du Canada». 
précieux document a été colligé par Patenaude lui-même et les éléments qu'il présente constituent, en grande partie, des renseignements qui sont ici publiés pour la première fois.

Ces notes identifiées «dossier personnel», semblent avoir été transcrites quelques années après les événements ${ }^{63}$. On pourrait alors douter de leur précision, Patenaude tentant de redorer son blason $a$ posteriori. Il faut certes les aborder avec cet esprit critique; elles gagnent cependant en crédibilité lorsque, confrontées avec d'autres écrits vraisemblablement inconnus de Patenaude, on constate une concordance quasi parfaite des éléments. Mieux, elles apportent parfois les liens unissant des bribes d'information ou d'analyse par ailleurs disparates.

Ainsi, le premier élément retracé dans ces notes nous permet de situer l'action des trois ministres par rapport à leur conviction idéologique, nous confirmant ce que nous avions pressenti: l'attitude de Casgrain, Blondin et Patenaude s'inspire nettement du credo nationaliste de 1911, les trois politiciens professant une foi inébranlable en un Canada uni composé de deux peuples fondateurs égaux. Le but qu'ils poursuivent vise à «faire définir le statut des Français au pays et probablement amener en même temps une nouvelle orientation politique du pays au sujet des relations entre les races, suivant l'esprit même de la Confédération et de ses auteurs ${ }^{64}{ }^{7}$. Selon son propre témoignage, Patenaude aurait lui-même insisté jusqu'à la dernière minute auprès du premier ministre en évoquant ces arguments précis:

Je lui représente que notre projet était sain et qu'il était lui-même dans l'erreur s'il laissait ainsi passer une occasion unique d'établir un règne d'entente et de paix entre les deux grandes races du pays [...] C'était d'ailleurs conforme au dire [désir?] des Pères de la Confédération que de vouloir interpréter l'acte constitutionnel de 1867, non pas en le resserrant dans des textes édroits [sic], secs et rigides mais en lui donnant un sens large et généreux suivant l'esprit qui avait animé les Pères de la Confédération dans le pacte qui avait alors été convenu entre les deux grandes races ${ }^{65}$.

Le doute, s'il devait subsister, n'est donc plus permis quant aux motifs de l'action des politiciens.

63. Le ton du document de même que quelques corrections manuscrites nous portent à le croire. Ébauche pour des mémoires que Patenaude n'a jamais complétées? Ce pourrait bien être le cas.

64. Idem, 1.

65. Idem, 5 . 
Par ailleurs, le document nous révèle aussi l'ampleur et le sérieux de la consultation préalable menée par les ministres avant qu'ils ne rédigent leur mémoire. Ceci constitue un élément important pour notre compréhension, car ill démontre l'importance que les trois hommes attachent à leur démarche: il ne s'agit pas d'un geste uniquement politique, il s'agit d'un geste relevant de convictions idéologiques. Patenaude nous révèle en effet que, en plus de Thomas Chapais, ses collègues et lui-même rencontrent le sénateur C. P. A. Beaubien et entretiennent une liaison constante avec l'un des principaux conseillers de Borden, Sir Charles Fitzpatrick, juge en chef de la Cour suprême et homme politique dont la notoriété en matière constitutionnelle est reconnue de tous. Comme le rappelle Patenaude:

C'est dans le plus grand secret que nous [l']avons approché [...] et que nous lui avons exposé notre projet d'intervention, lui demandant confidentiellement d'en faire l'étude et de nous donner son avis à ce sujet. Sir Charles fit bon accueil à notre proposition et nous a promis de l'étudier. Il nous communiqua immédiatement qu'il avait l'intention de s'adjoindre monsieur [Edward Robert] Cameron, greffier de la Cour suprême, pour l'aider dans ses recherches, sachant, disait-il, qu'il avait beaucoup d'expérience de ce côté et qu'il pouvait compter sur sa discrétion absolue ${ }^{66}$.

Les contacts établis par les trois ministres ne se limitent pas à ces conseillers. Apparemment décidés à aller jusqu'à la limite du possible, Patenaude, Blondin et Casgrain auraient été prêts à remettre «tous trois, ensemble en même temps [leur] démission» et à «présenter [euxmêmes leur] démarche aux plus hautes autorités britanniques ${ }^{67} \gg$. Et dans le but de préparer le terrain en ce sens, Patenaude affirme: «Nous avions même fait approcher des hommes d'autorité en France qui nous avaient promis d'appuyer notre demande auprès du gouvernement anglais et cela par l'entremise du gouvernement français ou de quelques-uns de ses représentants ${ }^{68} .{ }^{\prime}$

Face à une telle détermination, comment expliquer alors le recul des trois ministres et leur abdication finale? Patenaude attribue l'échec du projet à l'intervention du gouverneur général auprès de Casgrain.

À la suite de cette visite, écrit Patenaude, monsieur Casgrain m'appela pour me faire part de sa visite chez le gouverneur général, des représentations de ce dernier et surtout de ses ins-

66. Idem, 2.

67. Idem, 3 .

68. Idem. 
tances en vue d'obtenir de lui qu'il restât dans le ministère et qu'il abandonnât le projet que nous avions conçu et promis d'exécuter ensemble. Notre conversation se prolongea et il me déclara, en définitive, qu'il ne pouvait pas donner suite à notre projet et qu'il l'abandonnait définitivement. [...] Monsieur Casgrain me communiqua au cours des conversations qui suivirent qu'il maintenait sa décision ${ }^{69}$.

Puis, au sujet du secrétaire d'État, Patenaude tire les conclusions suivantes: «Que s'est-il passé avec M. Blondin, mon autre collègue? Je n'en sais rien. J'ai essayé pendant plusieurs jours de communiquer avec lui et de savoir ce qu'il avait décidé de faire, mais ce fut en vain. Je ne pouvais le rejoindre. J'ai compris cependant qu'il avait dû changer d'avis et abandonner à son tour notre projet ${ }^{70}$.»

Jusqu'où la solidarité de Patenaude envers ses deux collègues irat-elle? Qu'est-ce qui motivera son attitude puis sa prise de position définitive? La première réaction du ministre du Revenu en est une d'espoir: «croyant toujours que messieurs Casgrain et Blondin pourraient revenir sur leur décision et entreprendre d'exécuter le projet que nous avions formé ${ }^{71} \gg$. Constatant que l'un et l'autre demeurent sur ses positions, Patenaude leur affirme «qu'ils avaient tort d'abandonner ainsi notre projet ${ }^{72} \gg$. Patenaude, quant à lui, décide de poursuivre les démarches, ne retournant pas au Conseil des ministres, même si ses deux collègues y avaient regagné leur fauteuil. «J'ai ressenti une grande peine de cet abandon ${ }^{73} »$, confessera-t-il.

Le député d'Hochelaga se rend de nouveau chez le premier ministre tentant, une fois de plus, de le sensibiliser: il lui fait miroiter que le plan qu'ils avaient conçu pourrait «favoriser même l'effort de guerre auquelle [sic] il tenait par-dessus tout ${ }^{74} \gg$. Borden réfute tous les arguments de son ministre qui se retire pour réfléchir à la situation. «Je savais bien, écrit Patenaude, que je ne pourrais plus le convaincre d'adopter mon point de vue, surtout après l'abandon de mes collègues $^{75} . »$ Que fera alors le politicien?

Laissé seul, je ne pouvais pas aller plus loin. J'ai alors pensé à donner quand même ma démission mais il fallait dévoiler le secret de notre correspondance, communiquer au public le refus du premier ministre et de son gouvernement d'acquiescer à notre

\footnotetext{
69. Idem, 4

70. Idem.

71. Idem.

72. Idem.

73. Idem.

74. Idem.

75. Idem, 6 .
} 
demande et l'abandon, en cours de route, de notre projet par mes deux collègues. C'était évidemment précipiter une crise, activer l'agitation et rendre notre situation encore pire. [...] Aussi, sans rien abandonner de mes convictions et de mon attitude, j'ai décidé de reprendre mon siège au Conseil et de poursuivre mon travail et mes efforts ${ }^{76}$.

Cette dernière phrase nous laisse supposer que Patenaude est encore animé par ses convictions nationalistes. Son refus de démissionner pourrait pourtant nous laisser croire qu'il a succombé aux exigences de la lutte politique et qu'en réalité, il a enfoui ses convictions sous un opportunisme de mauvais aloi. Selon le principal intéressé, ce n'est toutefois pas le cas: d'une part, il insiste sur les nombreuses hésitations qu'il a eues avant d'acquiescer à la demande de Borden de revenir siéger au Cabinet; d'autre part, Patenaude nous donne les raisons profondes qui l'ont fait changer d'avis et adopter une position plus conciliante:

Personnellement, j'aurais mieux aimé démissionner, mais je savais que, dans ces conditions, cela ne guérirait pas et même ne diminuerait pas le mal profond dont l'élément français et le pays surtout souffraient. J'étais même convaincu qu'il valait mieux attendre une occasion plus favorable de faire valoir nos représentations et de reprendre le projet que nous avions formé ${ }^{77}$.

Ses principes nationalistes d'unité nationale, d'harmonie et de respect des races prenant nettement le dessus, il ajoute qu' «En définitive, j'ai réalisé de nouveau que ma démission ne pouvait pas rendre service aux miens et qu'elle pouvait, au contraire, leur nuire en faisant intensifier de part et d'autre l'agitation qui se poursuivait au pays ${ }^{78}$.»

Cette dernière partie du témoignage de Patenaude, celle où il explique lui-même sa prise de position, est sans doute celle envers laquelle nous sommes portés à être le plus critique: le politicien peut en effet nous servir le témoignage qu'il veut bien et peut l'agrémenter selon son bon désir. Il appert toutefois que, confronté au témoignage contenu dans le journal personnel de Borden, les propos de Patenaude et le climat qui s'en dégage semblent correspondre sur plusieurs points, sinon tous, avec ceux énoncés par le premier ministre.

Les écrits du premier ministre nous confirment, en effet, qu'à deux reprises et à la suite du changement d'attitude de Casgrain, Borden et Patenaude ont eu des entretiens ${ }^{79}$, pourparlers au cours

76. Idem, 6-7.

77. Idem, 6.

78. Idem, 7.

79. APC, Fonds Borden, Journal personnel, 26 et 27 avril 1916. 
desquels le député d'Hochelaga aurait affirmé à son chef: «I had decided to stay out of personal consideration for [him] and in view of the country's condition, but desire to retire from public life at first opportunity ${ }^{80} . »$ Quant à l'atmosphère pathétique qui semblait avoir plané sur ces journées d'intenses émotions, Borden le confirme en écrivant que Patenaude, seul et désarmé, devant abdiquer, «broke and wept ${ }^{81}$ ».

\section{ÉPILOGUE}

Concernant les efforts que Patenaude entend poursuivre en demeurant en politique active, s'agit-il là de quelque chose de concret ou sont-ce des vœux pieux? On peut être porté à croire que Patenaude est sincère, car il semble faire l'objet d'une certaine consultation, notamment de la part du ministre ontarien de l'Instruction publique, George Howard Ferguson. S'adressant à son collègue fédéral, celui-ci affirme: «There is not a shade of doubt in my mind that the agitation of interested parties has so befogged the real situation that the reasonable people have been misled into an entirely erroneous view of the whole matter. My whole anxiety and purpose is to provide and find a happy solution ${ }^{82}$.» Patenaude trouve enfin un écho à son appel à la fermeté dans la modération. "I cannot but agree with you in your contention, écrit-il en réponse à Ferguson, that grave misunderstanding exist to the true situation of affairs ${ }^{83}$.» Et les deux hommes d'entreprendre ainsi une période de pourparlers ${ }^{84}$. Ceux-ci ont-ils conduits directement ou indirectement à l'abrogation du Règlement XVII en 1927, alors que Ferguson occupe les fonctions de premier ministre ontarien? Bien que nos recherches n'aient pas fourni les indices nécessaires pour conclure à ce sujet, il pourrait s'agir d'une suite logique des événements. À moins que l'on ne recourt à la théorie de la simple maturation politique de Ferguson, tel que le soutient Arthur Godbout ${ }^{85}$.

80. Idem, 27 avril 1916.

81. Idem.

82. APA, Fonds Patenaude, boîte I, chemise «École d'Ontario III», Ferguson à Patenaude, 14 septembre 1916.

83. APA, Fonds Patenaude, loc. cit., Patenaude à Ferguson, 16 septembre 1916.

84. Pour le contexte de ces échanges, on peut consulter Peter Oliver, G. Howard Ferguson: Ontario Tory (Toronto, University of Toronto Press [1977]), 74-76.

85. Arthur Godbout, Nos écoles franco-ontariennes. Histoire des écoles de langue française dans l'Ontario, des origines du système scolaire (184I) jusqu'à nos jours (Ottawa, Éditions de l'Université d'Ottawa, 1980), 72. L'auteur y spécifie qu'au moment de renverser la décision gouvernementale ontarienne quant au Règlement XVII, Ferguson est «non plus l'arriviste politique des années 1910-1920, mais un brave homme rompu à toutes les intrigues de coulisses de l'Assemblée législative; [il] savait parfaitement dépassée toute action de coercition tendant à imposer à quelque $10 \%$ de la population un mode d'éducation qu'un nombre d'anglophones réputés [...] condamnait maintenant comme inefficace, inadapté aux besoins du pays et source constante d'inutiles confrontations entre les deux principaux groupes ethniques du Canada». 
Cette simple explication, par sa candeur, nous semble moins convaincante. Celle de l'abandon pur et simple d'une politique pratiquement impossible à faire respecter nous semble beaucoup plus plausible.

Du côté fédéral, on peut être porté à croire que Borden «[who] never showed much sympathy for the Franco-Ontarian cause ${ }^{86} \gg$ a cru bon de ne pas sacrifier des appuis anglophones sûrs en Ontario et dans le reste du pays, au risque de ne rien gagner dans un Québec qui tend à demeurer un château fort libéral ${ }^{87}$. Pendant ce temps, Ésioff-Léon Patenaude réaffirmait sa croyance en un Canada uni, en ses deux peuples égaux collaborant à l'édification de ce pays dans l'esprit même des Pères de la Confédération.

Quant à la dimension purement politique de la question, Patenaude n'est pas empreint de son idéologie nationaliste au point d'en oublier la nécessité du pouvoir pour permettre aux idées de se concrétiser. Avant que le débat sur les écoles ontariennes ne soit clos, et en réponse au nouveau chef conservateur Arthur Meighen qui cherche à redorer l'image de son parti au Québec, Patenaude répond: «La clé de la situation est dans la solution de la question scolaire d'Ontario. Si le parti conservateur pouvait créer l'impression qu'il se rapproche pour ne plus s'en écarter des principes posés par les Pères de la Confédération, je crois que nous ferions là un grand $\mathrm{pas}^{88}{ }^{88}$

$* * *$

L'étude du comportement du gouvernement conservateur fédéral dans l'épisode des écoles d'Ontario, nous fait voir une équipe ministérielle déchirée, au bord de la crise. Si la question même «atteint en plein cœur la minorité de cette province ${ }^{89}{ }_{\gg}$, elle est tout aussi dure pour le gouvernement, et plus particulièrement pour les membres canadiens-français du Cabinet. Il est toutefois fort intéressant de remarquer que l'affirmation de la suprématie de l'autonomie provinciale défendue par certains à la suite de leur chef et la défense d'une intervention pour la protection de la minorité préconisée par d'autres, nous révèlent autant une communauté d'esprit que la scission apparente des positions. En effet, cette double position affichée par les ministres canadiens-français et leurs supporters se rattache à une

86. Peter Oliver, op. cit., 75 .

87. Théorie développée par Roger Graham dans Arthur Meighen, I: The Door of Opportunity ([Toronto], Clarke, Irwin Co. Ltd., 1960), 102.

88. APC, Fonds Meighen, G. A. Fauteux à Meighen, 24 février 1925. Fauteux rapporte à son chef les paroles de Patenaude avec qui il a dîné ce midi-là.

89. Réal Bélanger cité par Gilles Gougeon, Histoire du nationalisme québécois. Entrevues avec sept spécialistes (Montréal, VLB éditeur/Société Radio-Canada, 1993), 63. 
même souche, à la racine même du nationalisme qu'ils préconisent à l'époque: soit la volonté que soit affirmé, voire affermi, l'esprit d'entente entre «les deux races» ayant animé les Pères de la Confédération. Plus particulièrement, le retour de Patenaude au Cabinet et son appel à la modération y trouvent leur explication. Face à ce nationalisme affirmé avec vigueur, joint à celui impérialiste, anglo-saxon et protestant préconisé par le reste du pays, le premier ministre Borden aura su tirer son épingle du jeu en fin politique qu'il était: il a répondu aux attentes de la majorité, se justifiant avec des arguments que la minorité ne pouvait récuser publiquement.

Notre réflexion nous aura donc permis de comprendre un peu mieux comment le délicat équilibre d'un Cabinet en crise et face à une crise a pu être maintenu. Mais loin d'apporter un point final à cette question, nous avons le sentiment d'avoir ouvert beaucoup plus de portes que nous n'en avons fermées. Ainsi, plusieurs questions demeurent sans réponse: quelle fut l'influence des ministres conservateurs fédéraux dans la décision de leur collègue ontarien Ferguson d'abroger, une fois devenu premier ministre provincial, le règlement honni? Quel rôle politique le gouvernement du Québec a-t-il joué dans ces événements? Qui étaient ces gens et quel était l'ampleur des consultations et des «approches» menées outre-Atlantique? Voilà bien des sentiers à explorer pour les collègues qui s'intéresseront à cette question. 